\title{
The 3-Hydroxy-3-Methylglutaryl-CoA Reductase Inhibitor Lovastatin Reduces Severity of L-DOPA-Induced Abnormal Involuntary Movements in Experimental Parkinson's Disease
}

\author{
Stefan Schuster, ${ }^{1}$ Agnès Nadjar, ${ }^{2}$ Jun Tang Guo, ${ }^{3}$ Qin Li, ${ }^{3}$ Carina Ittrich, ${ }^{1}$ Bastian Hengerer, ${ }^{1}$ and Erwan Bezard ${ }^{2}$ \\ ${ }^{1}$ Boehringer Ingelheim Pharma GmbH, 88397 Biberach, Germany, ${ }^{2}$ Centre National de la Recherche Scientifique, Unité Mixte de Recherche 5227, Universite \\ Victor Segalen-Bordeaux 2, 33076 Bordeaux, France, and ${ }^{3}$ Institute of Laboratory Animal Science, Chinese Academy of Medical Sciences, 100021 Beijing, \\ China
}

Chronic L-3,4-dihydroxyphenylalanine (L-DOPA) treatment of Parkinson's disease (PD) often leads to debilitating involuntary movements, termed L-DOPA-induced dyskinesia (LID), about which the rodent analog, the abnormal involuntary movements (AIMs), has been associated consistently with an activation of the Ras-extracellular signal-regulated kinase 1/2 (ERK1/2) mitogen-activated protein kinase signaling pathway. Previous studies have shown that lovastatin, a specific inhibitor of the rate-limiting enzyme in cholesterol biosynthesis, can also inhibit Ras isoprenylation and activity and subsequently the phosphorylation of ERK1/2 (pERK1/2). We hypothesized that lovastatin treatment-commenced previous L-DOPA exposure could reduce AIM incidence and severity in the 6-hydroxydopamine (6-OHDA) rat model of PD by secondarily preventing the $\mathrm{L}$-DOPA/Benserazide-induced increase in pERK1 levels. The lovastatin-L-DOPA/Benserazide-treated 6-OHDA animals displayed less severe rotational behavior as well as a dramatic reduction in AIM severity than the L-DOPA/Benserazide-treated ones. Such lower AIM severity was associated with a decrease in L-DOPA-induced increase in the following: (1) striatal pERK1 and (2) $\Delta$ FosB levels, and (3) theta/ $\alpha$ oscillations of substantia nigra pas reticulata (SNr) neurons as well as (4) a normalization of $\mathrm{SNr}$ firing frequency. Those results strongly suggest that lovastatin might represent a treatment option for managing LID in PD.

Key words: Parkinson's disease; statins; dyskinesia; AIMs; LID; 6-hydroxydopamine; substantia nigra pars reticulata; pERK1/2

\section{Introduction}

Chronic dopamine (DA) replacement therapy with L-3,4dihydroxyphenylalanine (L-DOPA) in Parkinson's disease (PD) results in motor complications known as L-DOPA-induced dyskinesia (LID) (Rascol, 2000). LID is associated with a sequence of events that include pulsatile stimulation of DA receptors, downstream changes in proteins and genes, and abnormalities in nonDAergic transmitter systems, all of which combine to produce alterations in the neuronal firing patterns that signal between the basal ganglia and the cortex (Bezard et al., 2001a). The rodent analog abnormal involuntary movements (AIMs) have been associated consistently with an activation of the Ras-extracellular signal-regulated kinase 1/2 (ERK1/2) mitogen-activated protein kinase (MAPK) signaling pathway (Gerfen et al., 2002; Westin et al., 2007). The conclusion of the study by Gerfen et al. (2002) was that "inhibitors of MAPK signaling cascade that would block the

Received 0ct. 18, 2007; revised March 10, 2008; accepted March 11, 2008.

We are grateful for the excellent technical assistance of Sandra Dovero, Laura Cardoit, and Li Hao.

Correspondence should be addressed to Erwan Bezard, Centre National de la Recherche Scientifique, Unité Mixte

de Recherche 5227, Universite Bordeaux 2, 146 Rue Leo Saignat, 33076 Bordeaux, France. E-mail: Erwan.bezard@u-bordeaux2.fr.

A. Nadjar's present address: Centre National de la Recherche Scientifique, Unité Mixte de Recherche 5226, Universite Victor Segalen-Bordeaux 2, 33076 Bordeaux, France.

D01:10.1523/JNEUROSCI.4720-07.2008

Copyright $\odot 2008$ Society for Neuroscience $\quad$ 0270-6474/08/284311-06\$15.00/0 aberrant supersensitive response of direct striatal pathway neurons may provide a novel therapeutic adjunct to the use of L-DOPA in the treatment of PD."

Lovastatin, a typical statin, is a specific inhibitor of the ratelimiting enzyme in cholesterol biosynthesis [3-hydroxy-3methylglutaryl-Coenzyme A (HMG-CoA) reductase] that is used widely to treat hyperlipidemia in humans (Corvol et al., 2003). Interestingly, lovastatin can inhibit Ras isoprenylation and activity (Li et al., 2005) and subsequently the phosphorylation (activation) of ERK1/2 (pERK1/2) (Li et al., 2005). Because LID is associated precisely with an increased ERK1/2 phosphorylation, we hypothesized that lovastatin treatment could reduce AIMs in the 6-hydroxydopamine (6-OHDA) rat model of PD.

\section{Materials and Methods}

Animals. Male Wistar rats (Harlan, Winkelmann, The Netherlands; Chinese Academy of Medical Sciences, Beijing, China) weighing 280-320 g at the time of testing were housed in groups of five in Macrolon type III cages under standard laboratory conditions $\left(22 \pm 1 \mathrm{C}^{\circ}, 55 \pm 5 \%\right.$ of a relative humidity, and a $12 \mathrm{~h}$ light/dark cycle) with ad libitum access to food and water. Experiments were approved by the ethical committees of the regional council of Upper Swabia (Tübingen, Germany) and of the Chinese Academy of Medical Sciences and performed in accordance with European Communities Council [Directive of 24 November 1986 (86/ 609/EEC)] for care of laboratory animals.

6-OHDA lesion. Unilateral mesencephalic lesions were performed as 
described previously (Meissner et al., 2006). 6-OHDA.Br $(2.5 \mathrm{mg} / \mathrm{ml}$; Sigma-Aldrich, St. Louis, MO) was infused $(0.3 \mu \mathrm{l} / \mathrm{min})$ through two small burr holes in the skull into the right medial forebrain bundle $[2.5 \mu \mathrm{l}$ at anteroposterior (AP) $-4.4 \mathrm{~mm}$, mediolateral (ML) $+1.2 \mathrm{~mm}$, and dorsoventral (DV) $-7.8 \mathrm{~mm}$, relative to bregma and dura; and $2 \mu \mathrm{l}$ at AP $-4.0 \mathrm{~mm}, \mathrm{ML}+0.7 \mathrm{~mm}$, and $\mathrm{DV}-8.0 \mathrm{~mm}$ ) (Paxinos and Watson, 1986).

Drug treatments. From 8 d postsurgery onwards, rats were treated once daily with lovastatin at a dose $(10 \mathrm{mg} / \mathrm{kg}$, i.p.) known to reduce brain pERK1/2 levels, produce behavioral effects in a cognitive paradigm ( $\mathrm{Li}$ et al., 2005), match with human dosages (10-80 $\mathrm{mg}$ in the clinic), and enter brain with a brain/plasma ratio of three after $1 \mathrm{~h}$ (Zhang and Yang, 2007). In addition, $3 \mathrm{~d}$ later (i.e., $11 \mathrm{~d}$ postsurgery), rats were also treated with L-DOPA [6 mg/kg, per os (p.o.)] and Benserazide ( $15 \mathrm{mg} / \mathrm{kg}$, p.o.), $5 \mathrm{~h}$ after lovastatin, for inducing AIMs in the 6-OHDA lesioned rats (Cenci et al., 1998). The two control groups were treated with $0.9 \%$ saline or LDOPA/Benserazide alone. Animals were killed on day 21, $1 \mathrm{~h}$ after LDOPA/Benserazide or vehicle administration. A subset of each group received $0.1 \mathrm{mg} / \mathrm{kg}$ subcutaneously of apomorphine (Sigma-Aldrich) $24 \mathrm{~h}$ after the last L-DOPA/Benserazide administration and were placed into an automated Rotameter (TSE Systems, Bad Homburg, Germany). The number of contralateral turns was counted from 30 min starting 10 min after apomorphine injection (Meissner et al., 2006).

Behavioral analysis. Rats were observed daily by an experimentally blind investigator every 20 th minute at $20-140 \mathrm{~min}$ after the administration of L-DOPA/Benserazide for $10 \mathrm{~d}$. The number of complete rotations made during each 20 min period was counted (mean \pm SEM) (Meissner et al., 2006). AIMs were rated and classified into four subtypes (axial, limb, orolingual, and locomotive) in exact accordance to Cenci's methodology (Cenci et al., 1998). Each subtype was scored on a severity scale from 0 to 4 ( 0 , nonexisting; 1 , present during less than half of the observation time; 2, present during more than half of the observation time; 3 , continuous but interrupted by strong sensory stimuli; 4 , continuous, not interrupted by strong sensory stimuli).

Assessment of lesion. After the rats were killed, their brains were removed and frozen in a Cryobain. Sections of either 20 or $200 \mu \mathrm{m}$ were cryostat cut. Sections $20 \mu \mathrm{m}$ thick were used for DA transporter binding, whereas $200-\mu \mathrm{m}$-thick sections were used for the Western blotting experiments. DA transporter binding using $\left[{ }^{125} \mathrm{I}\right]-(\mathrm{E})-\mathrm{N}$-(3-iodoprop2-enyl)-2 $\beta$-carboxymethyl-3 $\beta$-( $4^{\prime}$-methylphenyl)-nortropane (PE2I; Chelatec, Nantes, France) was measured as described previously (Bezard et al., 2001b). Only animals showing a DA transporter binding reduced by $>95 \%$ were retained for final analysis (see Fig. 1, inset).

Western blotting procedure. Dorsal striata were bilaterally dissected from $200-\mu \mathrm{m}$-thick sections. Samples were individually sonicated in a lysis buffer containing $1 \%$ SDS. After $30 \mathrm{~min}$ in ice, samples were centrifuged at $14,000 \mathrm{rpm}$, at $4^{\circ} \mathrm{C}$, for $20 \mathrm{~min}$. Supernatants were collected and stored at $-80^{\circ} \mathrm{C}$ until quantification of the whole protein content by the bicinchoninic acid method (Smith et al., 1985). Samples containing 100 $\mu \mathrm{g}$ of proteins were heated to $100^{\circ} \mathrm{C}$ for $5 \mathrm{~min}$ and electrophoresed onto a $10 \%$ polyacrylamide gel. Proteins were blotted on polyvinylidene fluoride membranes (Immobilon P; Millipore, Paris, France) in Miniprotean III (Bio-Rad, Paris, France). Membranes were saturated for $1 \mathrm{~h}$ by incubation with $5 \%$ bovine serum albumin (BSA; Sigma-Aldrich) in TBS-Tween (Tris-HCl, pH 7.5, $100 \mathrm{~mm} \mathrm{NaCl}, 0.1 \%$ Tween 20) and incubated overnight with the primary rabbit polyclonal anti-phosphoERK1/2, anti-ERK1/2 (both from Cell Signaling Technology, Danvers, MA), or anti-FosB (Santa Cruz Biotechnology, Heidelberg, Germany) diluted in TBS-Tween $0.1 \%$ supplemented with $1 \%$ BSA. Membranes were then incubated for $2 \mathrm{~h}$ at room temperature with the secondary anti-rabbit (1:5000; Jackson ImmunoResearch, West Grove, PA) or donkey anti-goat (Interchim, Montlucon, France) horseradish peroxidase-coupled antibody that was diluted in TBS-Tween $0.1 \%$ and supplemented with $3 \%$ BSA. The complexes were detected with enhanced chemiluminescence reagents (GE Healthcare, Little Chalfont, Buckinghamshire, UK), and immunoblots were evaluated by integrating densitometry using GeneSnap and GeneTools (Chemigenius Gel Documentation System; Syngene, Frederick, MD).

Electrophysiological recordings. On day 21 after surgery, recordings were performed as described previously (Meissner et al., 2006) in the ipsilateral substantia nigra pars reticulata $(\mathrm{SNr})$ of urethane-anesthetized animals (urethane 20\%, $0.8 \mathrm{ml} / 100 \mathrm{~g}$; Sigma-Aldrich) with single-barrel glass microelectrodes filled with $2 \%$ pontamine sky blue dye in $2 \mathrm{M} \mathrm{NaCl}$ (impedances between 5 and $10 \mathrm{M} \Omega$ ). The signal was recorded by direct current and sampled at $40 \mathrm{kHz}$ by an analogical/digital interface (Powerlab $4 \mathrm{~s}$; ADInstruments, Canberra, Australia) and stored on computer for off-line analysis. After stabilization, signal was recorded for $2 \mathrm{~min}$ (baseline). L-DOPA/Benserazide was then administrated intraperitoneally, and recordings were made for 2 min every $10 \mathrm{~min}$ for $100 \mathrm{~min}$. Pontamine sky blue was then iontophoresed from the recording electrode to enable the histological localization of the electrode tip. Off-line analysis was performed with a customized program written using Matlab (V6.5; MathWorks, Natick, MA). The recorded signal was filtered with a second-order digital Butterworth bandpass filter $(0.3-3 \mathrm{kHz})$ to flatten the baseline of the signal. A threshold was positioned to detect spikes with a signal-to-noise ratio higher than three. Mean firing rate and firing pattern (regular/bursting/irregular) were calculated for each spike using a density histogram method as described previously (Boraud et al., 2001). To analyze the frequency domain of the possible oscillation of each neuron, we first computed the autocorrelogram functions (AC) (Perkel et al., 1975) with a window of $1000 \mathrm{~ms}$ and a bin width of $10 \mathrm{~ms}$. Power spectral density (PSD) of AC was then calculated for $256 \mathrm{~ms}$ windows, which were shifted for $100 \mathrm{~ms}$. The analyses were performed separately for each $2 \mathrm{~min}$ period of recording. For additional statistical analysis, the frequency range was divided into three bands, theta/ $\alpha(4-10 \mathrm{~Hz}), \beta(14-30 \mathrm{~Hz})$, and gamma $(>30 \mathrm{~Hz}$ ) (Brown et al., 2002). For each frequency band, the PSD was defined as the average PSD in that band.

Statistical analysis. An ANOVA with repeated measurements was first performed, including the factors treatment and observation day and the interaction between treatment and observation day as fix and the factor animal as random factor for the data of all experimental days 4-10. For the covariance matrix, a banded Toeplitz block diagonal structure with three bands was chosen. A closed testing procedure was applied to control the family-wise error rate at a level of 5\%. Western blot data were analyzed using Kruskal-Wallis nonparametric ANOVA followed by post hoc $t$ tests corrected for multiple comparisons by the method of Dunn. Nonparametric spearman correlation test was used for testing linear regression. For multiple comparisons of mean firing rates and PSD in the specific frequency range, a one-way ANOVA for repeated measures was applied to estimate overall significance within experimental groups followed by post hoc $t$ tests corrected for multiple comparisons by the method of Bonferroni. Comparisons of firing pattern were made using the frequency of distribution test $\left(\chi^{2}\right.$, respectively, $\mathrm{df}=2, p<0.05$ when $\chi^{2}=5.99$ and $\mathrm{df}=98 ; p<0.05$ when $\left.\chi^{2}>122.11\right)$. The statistical evaluation was prepared using the software package SAS version 8.2 (SAS Institute, Cary, NC) and S-PLUS 6.1 (Insightful, Seattle, WA).

\section{Results \\ Lovastatin reduces incidence of \\ L-DOPA/Benserazide-induced AIMs}

The lovastatin-L-DOPA/Benserazide 6-OHDA-lesioned animals showed dramatically reduced locomotive AIMs (Fig. $1 A, B$ ) as well as a significant $(p<0.001)$ reduction of overall AIM severity compared with the L-DOPA/Benserazide-treated 6-OHDAlesioned rats (Fig. 1C,D). Further detailing the subtypes of AIMs, both the axial (Fig. 1E,F) and oro-lingual (Fig. $1 I-J$ ) AIM subtypes were significantly $(p<0.001)$ reduced (Fig. $1 G, H)$ but not the limb ones (Fig. 1G,H) that were actually increased during the first hour. These differences between groups were consistently observed throughout the $10 \mathrm{~d}$ L-DOPA/Benserazide treatment period (supplemental Fig. 1, available at www.jneurosci.org as supplemental material).

In a subset of animals challenged with apomorphine $(0.1 \mathrm{mg} /$ $\mathrm{kg}$, s.c.), there was no difference between the control groups (vehicle-treated 6-OHDA, $216.0 \pm 25.1$; L-DOPA/Benserazidetreated 6-OHDA, $225.6 \pm 18.9 ; n=9$; mean \pm SEM) and the 

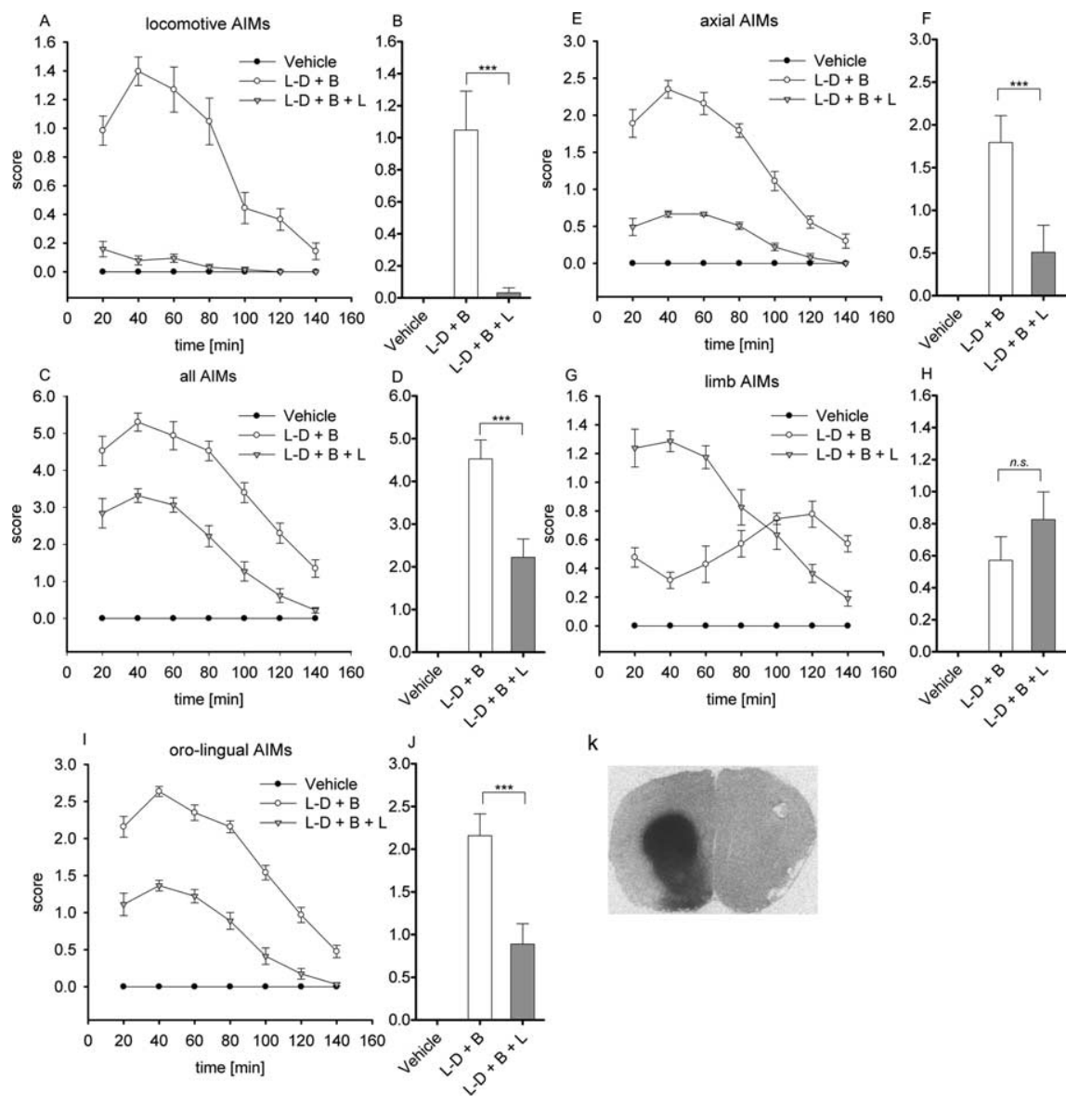

Figure 1. Effect of lovastatin on locomotive $(\boldsymbol{A}, \boldsymbol{B})$ and other AIMs $(\boldsymbol{C}-\boldsymbol{J})$ in the vehicle-treated 6-0HDA-lesioned rats $(n=9)$, L-DOPA (L-D)/Benserazide (B) 6-OHDA-lesioned rats $(n=9)$, and lovastatin (L)-L-DOPA/Benserazide 6-0HDA-lesioned rats $(n=$ 9). The inset in $\boldsymbol{A}$ shows a representative DAT binding autoradiogram featuring a $99 \%$ decrease on the lesioned (L) side versus the unlesioned side (UL). Cumulative AIM scores $(\boldsymbol{C}, \boldsymbol{D})$ were subdivised into axial $(\boldsymbol{E}, \boldsymbol{F})$, limb $(\boldsymbol{G}, \boldsymbol{H})$, and oro-lingual $(\boldsymbol{I}, \boldsymbol{J})$ subscores. The left panels show the time courses of behaviors monitored every $20 \mathrm{~min}$ over $140 \mathrm{~min}$. The right panels highlight the peak values $80 \mathrm{~min}$ after administration of L-DOPA/Benserazide. Data are mean \pm SEM of days $4-10$. Treatment effect is as follows: turn, $F_{(1,16)}=26.4, p<0.0001 ;$ all AlMs, $F_{(1,16)}=35.3, p<0.0001 ;$ axial, $F_{(1,16)}=22.3, p<0.001 ; \operatorname{limb}, F_{(1,16)}=2.5, p=$ 0.13 ; oro-lingual, $F_{(1,16)}=35.1, p<0.0001$. Observation day is as follows: turn, $F_{(6,96)}=1.7, p=0.13$; all AlMs, $F_{(6,96)}=0.69$, $p=0.66 ;$ axial, $F_{(6,96)}=0.18, p=0.98 ;$ limb, $F_{(6,96)}=0.65, p=0.69 ;$ oro-lingual, $F_{(6,96)}=0.65, p=0.69$. Interaction is as follows: turn, $F_{(6,96)}=1.48, p=0.19 ;$ all AlMs, $F_{(6,96)}=0.86, p=0.53 ;$ axial, $F_{(6,96)}=0.40, p=0.87 ; \operatorname{limb}, F_{(6,96)}=2.69, p<$ 0.05 ; oro-lingual, $F_{(6,96)}=0.76, p=0.60$.

lovastatin-L-DOPA/Benserazide 6-OHDA group $(208.1 \pm 28.4$; $\left.F_{(2,26)}=0.13 ; p=0.88\right)$ in a number of apomorphine-induced turns, suggesting that lovastatin does not interfere with the antiparkinsonian potency of dopaminomimetic therapy.

\section{Lovastatin reduces pERK1 and $\Delta$ FosB levels}

Our working hypothesis was that lovastatin treatment would secondarily decrease the L-DOPA/Benserazide-induced increase in pERK1 levels, and that this reduction would cause the reduction in AIM severity. The Western blotting experiment performed in the same three groups of animals clearly suggests that indeed lovastatin treatment significantly reduced pERK1 levels but not pERK2 levels (Fig. 2A) compared with the L-DOPA/Benserazide 6-OHDA-lesioned group back to control levels.

Because AIM development has been associated with $\Delta$ FosB induction (Andersson et al., 1999), we studied the transcription factor expression in each of the three conditions (Fig. $2 B$ ). LDOPA/Benserazide treatment induces a significant increase in $\Delta$ FosB levels as shown previously (Andersson et al., 1999), which is less important in lovastatin-treated animals (Fig. 2 B).

\section{Lovastatin normalizes AIM-linked SNr} pathological activity

Because we previously characterized the electrophysiological correlates of AIMs in this rodent analog of LID (Meissner et al., 2006), the electrophysiological activity of the output of the basal ganglia motor loop, the $\mathrm{SNr}$, was recorded before and after acute drug administration. The basal firing rate (at $t=0 \mathrm{~min}$ ) was comparable in the SNr neurons of the contralateral (17.9 \pm 0.6 spike/s) and ipsilateral $(18.0 \pm 0.7$ spike/s) side to the lesion in the vehicletreated 6-OHDA-lesioned rats. The basal firing rate was significantly increased in the L-DOPA/Benserazide 6-OHDAlesioned and lovastatin-L-DOPA/Benserazide 6-OHDA-lesioned rats compared with the vehicle-treated 6-OHDAlesioned rats $(p<0.05)$ (Fig. 3A). Although L-DOPA/Benserazide administration reduced $\mathrm{SNr}$ firing compared with baseline $(p<0.05)$ below control levels $(p<0.05)$ (Fig. 3A), lovastatin-L-DOPA/ Benserazide reduced it as well compared with baseline $(p<0.05)$ but was not any more different from control levels (Fig. 3A).

Although the $\mathrm{SNr}$ firing pattern was not affected by the treatments (Fig. 3B), the oscillation power calculated on the autocorrelogram was dramatically changed in the theta and low $\alpha$ range band (i.e., the $4-10 \mathrm{~Hz}$ band) (Fig. $3 C$ ) but not in the other bands (i.e., $\beta$ and gamma) (data not shown), as shown previously (Meissner et al., 2006). Although oscillation power in the theta/ $\alpha$ range was comparable at baseline between groups (Fig. $3 C$ ), it increased in the L-DOPA/Benserazide 6-OHDAlesioned rats as well as in the lovastatin-LDOPA/Benserazide 6-OHDA-lesioned rats after drug administrations $(p<0.05$ vs baseline in both groups) (Fig. $3 C$ ). However, the increase in the lovastatin-L-DOPA/Benserazide 6-OHDA-lesioned rats was more modest as the oscillation power was significantly lower than in the L-DOPA/Benserazide 6-OHDA-lesioned rats $(p<0.05)$ (Fig. 3C).

\section{Discussion}

The present study shows that cotreatment with lovastatin reduces the severity of L-DOPA/benserazide-induced rotational behavior and AIMs. Lower AIM severity was associated with a decrease in L-DOPA-induced increase in (1) striatal pERK1 and (2) $\Delta$ FosB levels, and (3) theta/ $\alpha$ oscillations of SNr neurons as well as (4) a normalization of SNr firing frequency. Those results strongly suggest that lovastatin might represent a treatment option for managing LID in PD.

\section{Specific AIM subtypes are improved}

Chronic cotreatment with lovastatin reduced the severity of L-DOPA/benserazide-induced rotational behavior and AIMs (Fig. 1) over the experimental period. The reduction in turning 

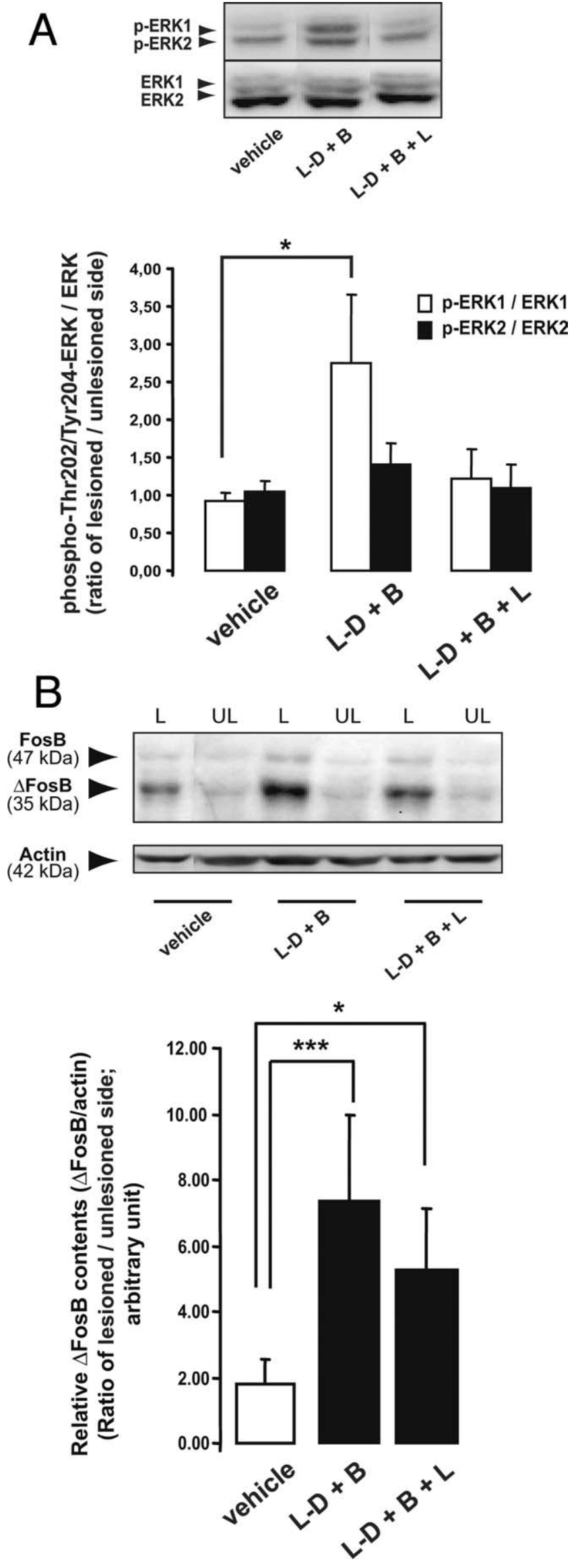

behavior (Fig. 1A) was not associated with a decreased antiparkinsonian response to L-DOPA/Benserazide, because all groups responded comparably to apomorphine challenge. The fact that they were still displaying some AIMs (Fig. 1C), such as the limb subtype (Fig. 1G), strongly suggests the sensitized response was maintained in specific basal ganglia subnetworks responsible for the expression of those AIMs but the pathological sensitized behavior had decreased. When considered altogether, all AIMs were significantly decreased by $51.4 \%$, a magnitude of effect that is highly clinically relevant. Although both the orolingual and axial AIMs were dramatically decreased, the limb subtype was not and was even worsened during the first hour after L-DOPA/Benserazide administration (Fig. 1G). Interestingly, choreic-like LID/AIMs are thought to generally involve overactivity of the direct striatal output pathway and that LID/ AIMs characterized by dystonia might additionally involve the motor components of the indirect striatal output pathway (Bezard et al., 2001a). The limb subtype of AIMs belong to the choreic class of abnormal movements (Cenci et al., 2002) and would therefore primarily involve the direct pathway medium-spiny neurons that coexpress the $\mathrm{DA} \mathrm{D}_{1}$ receptor and dynorphin.

\section{AIM improvement correlates with reduction in striatal pERK1 levels}

Increased signaling through $\mathrm{DA} \mathrm{D}_{1}$ receptors is implicated in the abnormal regulation of molecular and synaptic responses in striatal neurons at the heart of LID (Picconi et al., 2003). L-DOPA treatment causes phosphorylation of ERK1/2 in the DAdenervated striatum after acute and chronic administration in both enkephalin-positive and dynorphin-positive neurons (Westin et al., 2007) through a DA $D_{1}$-receptor-dependent mechanism (Gerfen et al., 2002; Westin et al., 2007). Such an effect would be mediated through direct action on DA $\mathrm{D}_{1}$ receptors expressed by direct pathway medium-spiny neurons and through secondary indirect activation of the basal ganglia-thalamocortico-basal ganglia network on indirect pathway mediumspiny neurons expressing DA $\mathrm{D}_{2}$ receptor (Westin et al., 2007). Similar to previous reports (Gerfen et al., 2002; Westin et al., 2007), pERK1 and $\triangle$ FosB levels correlated with AIM severity, with increased levels in L-DOPA/Benserazide 6-OHDA-lesioned rats and decreased levels in lovastatin-L-DOPA/Benserazide 6-OHDA-lesioned animals (Fig. 2). The fact that lovastatin acts independently of DA receptors leaves open the possibility of a differential involvement of the direct versus indirect pathway medium-spiny neurons in mediating its effects. Because of the limb AIMs, one can therefore hypothesize that lovastatin treat-

$\leftarrow$

Figure 2. $\quad A$, Effect of lovastatin $(L)$ on striatal pERK1/ERK1 and pERK2/ERK2 protein levels detected by Western blot in the vehicle-treated 6-OHDA-lesioned rats $(n=8), \mathrm{L}-\mathrm{DOPA}(\mathrm{L}-\mathrm{D}) /$ Benserazide (B) 6-OHDA-lesioned rats $(n=7)$, and lovastatin-L-DOPA/Benserazide 6-OHDAlesioned rats $(n=7)$. The top of the figure shows the actual Western blot results. Quantifications are summarized in the graph below. Data (mean \pm SEM) are expressed as the ratio of pERK versus total ERK (ERK antibody recognizes phosphorylated and nonphosphorylated form of ERK) from the lesioned side divided by the same ratio from the unlesioned side (each rat is its own control). ${ }^{*} p<0.05$ versus saline-treated control group. $B$, Effect of lovastatin treatment on $\triangle$ FosB expression levels in the vehicle-treated 6-OHDA-lesioned rats $(n=6)$, L-DOPA/ Benserazide 6-0HDA-lesioned rats $(n=8)$, and lovastatin-L-DOPA/Benserazide 6-OHDAlesioned rats $(n=8)$. The top of the figure shows the actual Western blot results. Quantifications are summarized in the graph below. Data (mean \pm SEM) are expressed as the ratio of $\triangle$ FosB versus actin levels (loading control) from the lesioned side divided by the mean value of $\Delta$ FosB/actin ratio in the unlesioned side. L-DOPA-treated versus 6-0HDA-treated, ${ }^{* * *} p<$ 0.001; lovastatin-treated versus 6-OHDA-treated, ${ }^{*} p<0.05$. 
A

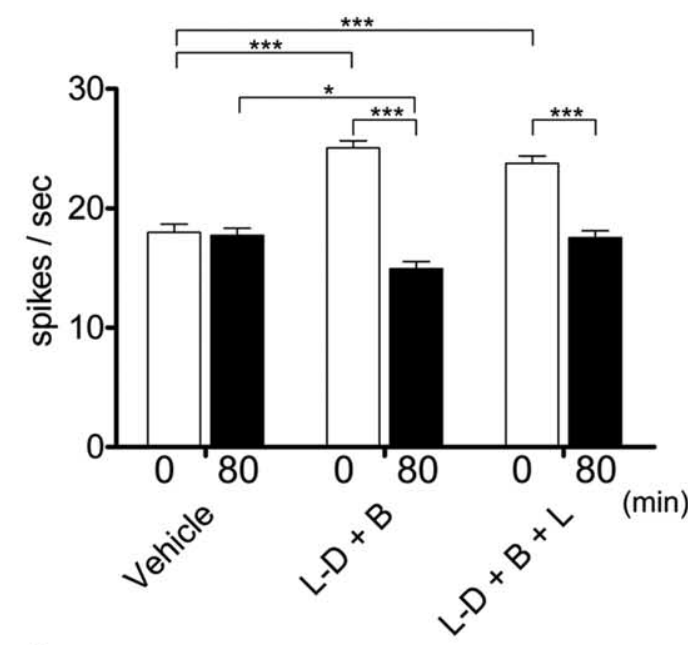

B

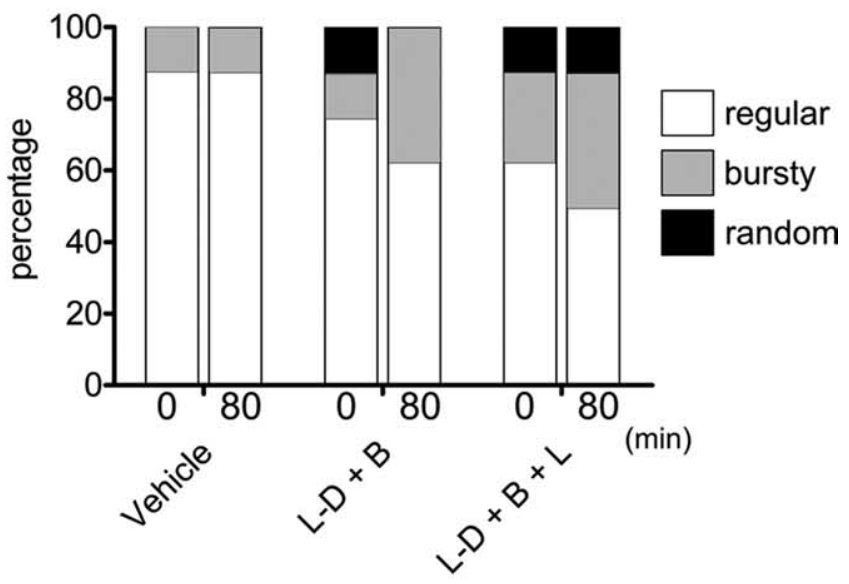

C Oscillations

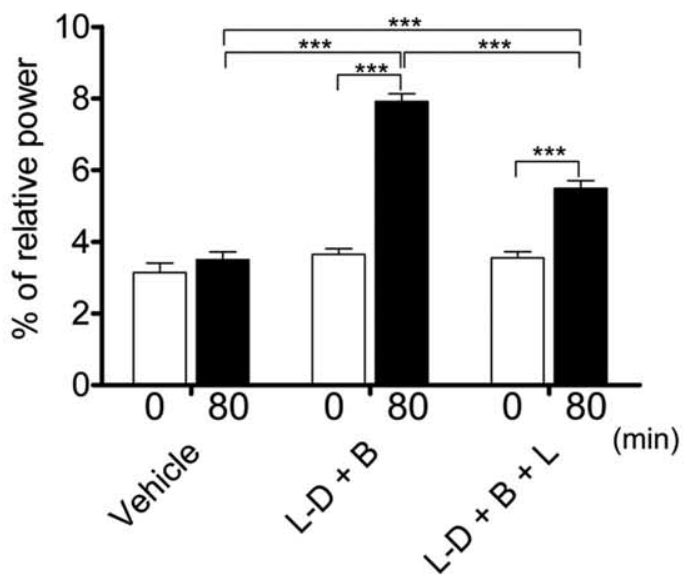

Figure 3. Effect of lovastatin $(L)$ on $\mathrm{SNr}$ neuron electrophysiological activity in the vehicletreated 6-OHDA-lesioned rats $(n=8)$, L-DOPA (L-D)/Benserazide (B) 6-OHDA-lesioned rats $(n=7)$, and lovastatin-L-DOPA/Benserazide 6-OHDA-lesioned rats $(n=7)$. Because one neuron was recorded per rat, $n$ of neurons equates $n$ of animals. $A$, Single-unit mean firing rate (mean \pm SEM). Group effect, $F_{(2,47)}=13.2, p<0.0001$; time effect, $F_{(1,47)}=127.8, p<$ 0.0001; interaction, $F_{(2,47)}=35.3, p<0.0001$. B, Pattern of discharge (percentage of each mode of discharge). $C$, Mean oscillation power in the theta $\alpha$ range (4-10 Hz; mean $\pm S E M)$. Group effect, $F_{(2,35)}=70.2, p<0.0001$; time effect, $F_{(1,35)}=166.2, p<0.0001$; interaction, $F_{(2,35)}=44.6, p<0.0001 .{ }^{*} p<0.05$, and ${ }^{* * *} p<0.001$. ment better normalizes pERK1 levels in indirect pathway than in direct pathway medium-spiny neurons, that this phenomenon is direct (action at striatum level) or indirect (normalization of corticostriatal activity).

\section{AIM improvement correlates with $\mathrm{SNr}$ firing rate and oscillation in theta/ $\alpha$ range}

A key add on of this study is to report the changes in electrophysiological activity of an output structure of the basal ganglia, the SNr. Previous studies have indeed shown that dyskinesia manifestations correlate with an overdecreased firing frequency of the basal ganglia output structures in the rat (Meissner et al., 2006) and nonhuman primate models of LID (Boraud et al., 2001) as well as in dyskinetic PD patients (Hutchinson et al., 1997). Moreover, the role of synchronized activity among neuronal populations in the theta $/ \alpha$ band has been acknowledged in the AIM rat model (Meissner et al., 2006) and in PD patients (Alonso-Frech et al., 2006). Indeed, those studies showed a correlation between local field potential (LFP) power in the theta/ $\alpha$ band in the subthalamic nucleus (Alonso-Frech et al., 2006) and SNr (Meissner et al., 2006) and the severity of LID and AIMs, respectively. In addition Meissner et al. (2006) demonstrated that SNr activity at single-cell level was likely driven by LFP incoming activity, because it oscillated in the theta $/ \alpha$ range as well in direct correlation with AIMs. Here, for the first time, we used these criterions for assessing the potential of an antidyskinetic strategy. LovastatinL-DOPA/Benserazide 6-OHDA-lesioned animals displayed $\mathrm{SNr}$ neuronal firing frequency (Fig. $3 A$ ) comparable with controls and decreased oscillation power in the theta/ $\alpha$ range compared with the L-DOPA/Benserazide 6-OHDA-lesioned rats (Fig. 3B). Interestingly, the lack of complete normalization of theta/ $\alpha$ oscillation power correlates with the remaining AIM manifestations in those animals.

\section{Conclusion}

Previous studies have shown that an increase in pERK1 activity is central to the pathophysiology associated with dyskinesia (Gerfen et al., 2002; Westin et al., 2007). Our behavioral, biochemical, and electrophysiological data demonstrate that lovastatin reduces AIM severity, reverses the abnormally elevated pERK1 and $\Delta$ FosB levels, and normalizes electrophysiological indexes associated with AIMs in an animal model of LID. Although lovastatin is a specific inhibitor of HMG-CoA reductase, interaction with other pathways related to inhibition of mevalonate (its product) or isoprenoids and the metabolic pathways of L-DOPA decreasing dopamine effects cannot be excluded. To this end, lovastatin treatments combined with $\mathrm{DA} \mathrm{D}_{1}$ and $\mathrm{D}_{2}$ agonists should now be tested together with additional studies aiming at dissecting out the exact mechanism of action on corticobasal ganglia network and for refining the magnitude of effect on the variety of behavioral manifestations. Altogether, the studies reported here demonstrate that AIM manifestations can be reversed by treatments with lovastatin, a widely prescribed drug that is known to be well tolerated even in long-term treatments. Therefore, these data suggest that lovastatin could be used to treat LID humans.

\section{References}

Alonso-Frech F, Zamarbide I, Alegre M, Rodriguez-Oroz MC, Guridi J, Manrique M, Valencia M, Artieda J, Obeso JA (2006) Slow oscillatory activity and levodopa-induced dyskinesias in Parkinson's disease. Brain 129:1748-1757.

Andersson M, Hilbertson A, Cenci MA (1999) Striatal fosB expression is causally linked with L-DOPA-induced abnormal involuntary movements 
and the associated upregulation of striatal prodynorphin mRNA in a rat model of Parkinson's disease. Neurobiol Dis 6:461-474.

Bezard E, Brotchie JM, Gross CE (2001a) Pathophysiology of levodopainduced dyskinesia: potential for new therapies. Nat Rev Neurosci 2:577-588.

Bezard E, Dovero S, Prunier C, Ravenscroft P, Chalon S, Guilloteau D, Crossman AR, Bioulac B, Brotchie JM, Gross CE (2001b) Relationship between the appearance of symptoms and the level of nigrostriatal degeneration in a progressive 1-methyl-4-phenyl-1,2,3,6-tetrahydropyridinelesioned macaque model of Parkinson's disease. J Neurosci 21: 6853-6861.

Boraud T, Bezard E, Bioulac B, Gross CE (2001) Dopamine agonist-induced dyskinesias are correlated to both firing pattern and frequency alterations of pallidal neurones in the MPTP-treated monkey. Brain 124:546-557.

Brown P, Kupsch A, Magill PJ, Sharott A, Harnack D, Meissner W (2002) Oscillatory local field potentials recorded from the subthalamic nucleus of the alert rat. Exp Neurol 177:581-585.

Cenci MA, Lee CS, Bjorklund A (1998) L-DOPA-induced dyskinesia in the rat is associated with striatal overexpression of prodynorphin- and glutamic acid decarboxylase mRNA. Eur J Neurosci 10:2694-2706.

Cenci MA, Whishaw IQ, Schallert T (2002) Animal models of neurological deficits: how relevant is the rat? Nat Rev Neurosci 3:574-579.

Corvol JC, Bouzamondo A, Sirol M, Hulot JS, Sanchez P, Lechat P (2003) Differential effects of lipid-lowering therapies on stroke prevention: a meta-analysis of randomized trials. Arch Intern Med 163:669-676.

Gerfen CR, Miyachi S, Paletzki R, Brown P (2002) $D_{1}$ dopamine receptor supersensitivity in the dopamine-depleted striatum results from a switch in the regulation of ERK1/2/MAP kinase. J Neurosci 22:5042-5054.

Hutchinson WD, Levy R, Dostrovsky JO, Lozano AM, Lang AE (1997) Ef- fects of apomorphine on globus pallidus neurons in parkinsonian patients. Ann Neurol 42:767-775.

Li W, Cui Y, Kushner SA, Brown RA, Jentsch JD, Frankland PW, Cannon TD, Silva AJ (2005) The HMG-CoA reductase inhibitor lovastatin reverses the learning and attention deficits in a mouse model of neurofibromatosis type 1. Curr Biol 15:1961-1967.

Meissner W, Ravenscroft P, Reese R, Harnack D, Morgenstern R, Kupsch A, Klitgaard H, Bioulac B, Gross CE, Bezard E, Boraud T (2006) Increased slow oscillatory activity in substantia nigra pars reticulata triggers abnormal involuntary movements in the 6-OHDA-lesioned rat in the presence of excessive extracellular striatal dopamine. Neurobiol Dis 22:586-598.

Paxinos G, Watson C (1986) The rat brain. San Diego: Academic.

Perkel DH, Gerstein GL, Smith MS, Tatton WG (1975) Nerve-impulse patterns: a quantitative display technique for three neurons. Brain Res 100:271-296.

Picconi B, Centonze D, Hakansson K, Bernardi G, Greengard P, Fisone G, Cenci MA, Calabresi P (2003) Loss of bidirectional striatal synaptic plasticity in L-DOPA-induced dyskinesia. Nat Neurosci 6:501-506.

Rascol O (2000) The pharmacological therapeutic management of levodopa-induced dyskinesias in patients with Parkinson's disease. J Neurol 247:II51-II57.

Smith PK, Krohn RI, Hermanson GT, Mallia AK, Gartner FH, Provenzano MD, Fujimoto EK, Goeke NM, Olson BJ, Klenk DC (1985) Measurement of protein using bicinchoninic acid. Anal Biochem 150:76-85.

Westin JE, Vercammen L, Strome EM, Konradi C, Cenci MA (2007) Spatiotemporal pattern of striatal ERK1/2 phosphorylation in a rat model of L-DOPA-induced dyskinesia and the role of dopamine $\mathrm{D}_{1}$ receptors. Biol Psychiatry 62:800-810.

Zhang Z, Yang Z (2007) HPLC determination of lovastatin in rat tissue. Chromatografia 66:487-491. 